\title{
Transport phenomena and modelling in melting and refining processes
}

\author{
D. ABLITZER
}

LSG2M/URA CNRS 159, Ecole des Mines de Nancy, Parc de Saurupt, 54042 Nancy cedex, France

\begin{abstract}
$\underline{\text { Abstract }}$
Increasing requirements for ultra pure metallic materials with greater reliability and more reproducible mechanical properties have led over the years to a continual endeavour to improve the control of composition and inclusion contents (nitrides, oxides, carbides, etc.) in cast products.

This is illustrated by melting and refining processes such as Vacuum Induction Melting (VIM), $\checkmark$ acuum Arc Remelting (VAR), Electro Slag Remelting (ESR), Electron Beam Melting (EBM) and Electron 3 eam Cold Hearth Remelting (EBCHR). The materials involved are nickel-base superalloys, specialty steels. refractory metals such as tantalum, niobium, tungsten and molybdenum, and the reactive metals hafnium, zirconium and titanium, together with their alloys.
\end{abstract}

The mastery of these refining processes requires a perfect knowledge of the physical-chemical phenomena involved, including both thermodynamic aspects and transport mechanisms within the liquid metal and at the interfaces (particularly that between the melt and the vacuum or gaseous phase), together with the behaviour of inclusions.

Examples are described to show how this knowledge of the basic phenomena can be combined with process modelling to improve the control of product quality.

\section{I - INTRODUCTION}

In the field of melting and remelting, the principal techniques concerned are the VIM (Vacuum Induction Melting), VAR (Vacuum Arc Remelting), EBM (Electron Beam Melting), and EBCHR (Electron Beam Cold Hearth Remelting) processes, and a number of others, such as Plasma Cold Hearth Remelting, Cold Crucible Induction Melting, etc.

A wide range of metals and alloys are concerned, but because of the high process costs, they are principally limited to high value added products, such as titanium and zirconium and their alloys, niobium, tantalum, the superalloys and numerous high performance nickel or iron base materials.

The phenomena involved in these melting and remelting techniques are both numerous and complex. The phenomena which must be considered are :

- the thermodynamic equilibria,

- the various transfer mechanisms within the liquids :

- the movement of the liquid metal, particularly with regard to electromagnetic stirring and turbulence,

- the movement of the slag,

- heat transfer processes,

- transfer of the different solute elements,

- the displacement of inclusions, 
- the transfer mechanisms at the various interfaces :

- liquid metal/vacuum (or gas),

- liquid metal/solid.

- liquid metal/inclusions,

- liquid metal/refractory,

- liquid metal/slag.

It must be remembered that a material is fabricated to fulfil specific functions, which require certain properties, themselves dependant on the composition and the structure of the material. In order to satisfy this double requisite "composition-structure", the complete melting, processing and heat treatment cycle must be controlled. Any non-compliance with specifications during melting cannot generally be corrected at a later stage, and will lead to a poor quality product.

Since it is impossible to give an exhaustive coverage, this paper will focus on the 4 remelting processes (VAR, ESR, EBM, EBCHR). Certain phenomena will be particularly emphasised in each of them.

\section{II - THE ROLE OF MATHEMATICAL MODELLING}

The melting and remelting processes are complex and are characterized by a number of features which make in-situ measurements difficult :

- high temperatures,

- the reactivity of the alloys,

- the important contribution of heterogeneous reactions.

In this context, mathematical modelling based on physical-chemical phenomena offers a means of investigation which is often irreplaceable, provided that it is closely associated with laboratory measurements and experimental validations. The capacity of present-day computers enables the treatment of ever more complex phenomena and geometries. Numerous calculation codes are commercially available, but the user can also choose to develop his own programs. The numerical methods employed are varied and powerful. Examples are the finite difference, finite volume and finite element techniques.

III - VACUUM ARC REMELTING : control of the structure and composition of the allovs produced: optimization of the operating conditions.

The VAR process is used for the production of special steels and nickel or cobalt base alloys for the aircraft industry. It also represents the final stage of the melting cycle for zirconium and titanium alloys.

Depending on the property it is wished to obtain in the product : solidification structure, chemical homogeneity, absence of micro-shrinkage, macro-segregations or pipes, it will be necessary to control different types of phenomena (thermal, hydrodynamic, matter transfer, interface reactions, etc.) (figure 1).

\section{1) Thermal modelling of the ingot: minimization of the local solidification time.}

A simple thermal model of the ingot enables the temperature distribution to be determined at any instant, thus indicating the position of the shrinkage pipe and allowing evaluation of the local solidification time (l.s.t.). The 1.s.t. is defined as the time taken for a given point in the ingot to cool from the liquidus temperature to the temperature corresponding to the end of solidification. It is directly related to structural parameters such as the interdendritic spacings. In general, the larger the l.s.t., the coarser the structure. Models of this type have been in use for many years.

These models have been validated by deliberately marking the liquid pool during remelting operations in both pilot and industrial furnaces.

With models of this sort, it is possible to determine the operating conditions which lead to a minimum local solidification time in a given ingot. This is done by calculating the variation of the l.s.t. 
under the last liquid pool as a function of the melting rate. The position of the minimum 1.s.t. indicates the optimum melting rate to be employed.

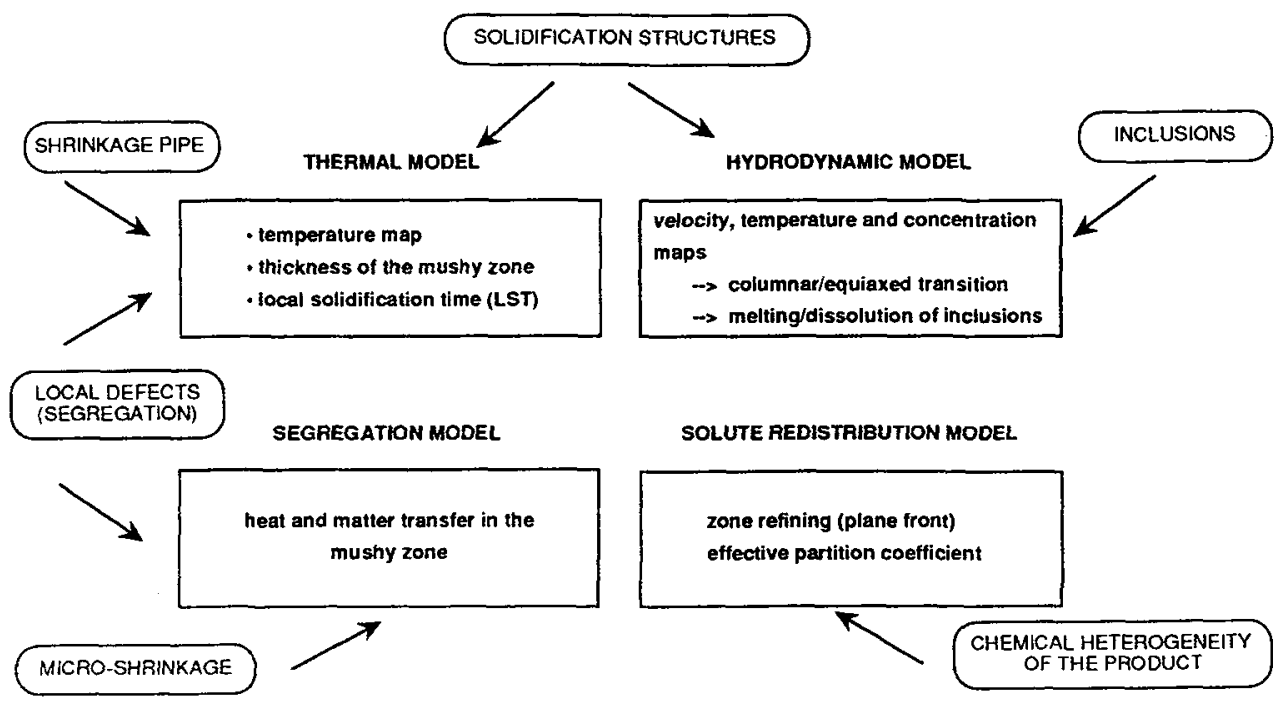

Figure 1 : Relationship between the product quality to be obtained and the modelling approach to be developped.

2) Reduction of longitudinal segregations : the case of oxygen in TA6V alloy.

The coexistence of the liquid and solid phases in the VAR process leads to a zone refining phenomenon. This causes axial segregation of the alloying elements on the scale of the ingot. For the titanium alloys used in aircraft engines, this segregation results in a scatter in the $\alpha-\beta$ transus temperature, which is strongly dependent on chemical composition. In order to control this critical parameter, the company Cezus, in collaboration with the Nancy School of Mines, developed a model for calculating the charge compensation required to minimise the final macrosegregation.

The model is of the two-dimensional "zone refining" type, in which:

- the solidification front is assumed to be locally planar. This assumption is sufficient for relatively lean alloys (e.g. Zy4, Zy2, TA6V). The front is then localized along a mean isotherm situated between the liquidus and the solidus. This isotherm is determined by the thermal model previously described;

- the melt pool is assumed to have a uniform composition, except for a diffusion layer along the solidification front;

- solute redistribution is characterized by an effective partition coefficient $\mathrm{K}_{\mathrm{eff}}$.

The solution of the solute conservation equation both for the solid phase and for the liquid phase, with the appropriate initial and boundary conditions, enables the variation of the ingot composition to be determined during successive remelting operations.

It is thus shows that, starting with a homogeneous TA6V ingot, after 3 successive melting runs, taking care to turn the ingot over each time, the final oxygen distribution obtained is not uniform.

The same calculation made for an electrode with initial compensation of the charge shows that it is possible to significantly reduce the heterogeneity. 


\section{3) Movement in the melt pool}

In order to calculate the movement of the liquid metal in the melt pool, it is necessary to take into account the interaction between thermal and hydrodynamic factors.

The heat transfer is represented by the heat balance :

$$
\rho \mathrm{C}_{\mathrm{p}} \overrightarrow{\mathrm{V}} \vec{\nabla} \mathrm{T}=\vec{\nabla}\left(\left(\lambda+\lambda_{\mathrm{t}}\right) \vec{\nabla} \mathrm{T}\right)+\mathrm{Q}
$$

where $C_{p}$ is the specific heat of the fluid, $\lambda$ is the thermal conductivity and $Q$ is the energy per unit volume dissipated by resistance heating.

The latent hegt transported by the liquid metal droplets is taken into account by the term $\rho \mathrm{C}_{\mathrm{p}} \overrightarrow{\mathrm{V}} \cdot \vec{\nabla} \mathrm{T}$, where the velocity $V$ represents the sum of the velocity of the liquid in the pool and the velocity of the droplets (advection). The turbulent thermal conductivity is calculated from the turbulent viscosity $\mu_{\mathrm{t}}$ by considering as a first approximation that :

$$
\mathrm{P}_{\mathrm{t}}=\mathrm{C}_{\mathrm{p}} \frac{\mu \mathrm{t}}{\lambda \mathrm{t}}=1
$$

The movements in the melt pool are represented by the momentum transfer equations for an incompressible fluid :

$$
\rho \vec{V} \cdot \vec{\nabla} \vec{V}=-\vec{\nabla} \mathrm{P}+\vec{\nabla}((\mu+\mu \mathrm{t}) \vec{\nabla} \overrightarrow{\mathrm{V}}) \overrightarrow{\mathrm{F}}
$$

where $P$ is the pressure, $\vec{V}$ is the velocity vector, $\rho$ is the density of the fluid, and $\mu$ is the dynamic viscosity.

The force vector per unit volume, $\vec{F}$, is equal to the sum of the gravitational and Lorentz forces :

$$
\vec{F}=\mu_{0} \vec{J} \wedge \vec{H}+\rho \vec{g}
$$

where $\vec{g}$ is the acceleration due to gravity.

The density $\rho$ can vary with temperature $T$ according to the linear law

$$
\rho=\rho_{0}\left(1-\beta\left(T-T_{0}\right)\right)
$$

where $\beta$ is the thermal expansion coefficient of the fluid.

This signifies that the Navier-Stokes, turbulence and energy conservation equations must be solved in an interactive manner. The results obtained, for a TA6V ingot, reveal the liquid metal recirculation vortices. These can be created by two types of volume phenomena : the divergence of the electric current flux lines and the natural convection due to the radial thermal gradients. These movements lead to a marked temperature stratification in the liquid pool.

However, in the VAR process, it is common practice to use an external coil to induce stirring in the melt pool. The liquid metal is then given a circular movement which can be either unidirectional or alternating. The solution of the magnetic field equations enables calculation of the force field applied to the liquid metal. Introduction of the latter in the Navier-Stokes equations enables calculation of the angular velocity of the metal. The results indicate that the angular velocity at the bottom of the pool is particularly low when alternating stirring is employed. 


\section{4) Overall model for the remelting of rich allovs}

For the remelting of rich alloys, it is necessary to take into account the transport equations in the mushy or pasty zone.

It is possible to develop an overall model taking into account all the preceding phenomena. It includes in the transport equations, in particular in the momentum conservation equation, a term reflecting the fact that the mushy zone is considered as a porous medium.

The hydrodynamics of the liquid metal and the temperature distribution throughout the ingot can be determined in the completely transient regime. It can be seen that the flow affects part of the mushy zone.

A model of this type is capable of predicting the segregations in the ingot, whatever the shape and thickness of the mushy zone.

\section{IV - ELECTRO SLAG REMELTING}

All which has been said about the VAR process can be applied to electro slag remelting. However, the Navier-Stokes, turbulence, heat transfer and solute transfer equations must also be solved in the slag.

For example, in the case of a chromium steel. Figure 2 shows the movements in the slag and the liquid pool. Three recirculation vortices can be seen in the slag : two are due to the predominance of natural onvection and the third is caused by the divergence of the electric current flux lines. The temperature distributions in the slag and the liquid metal can also be calculated.

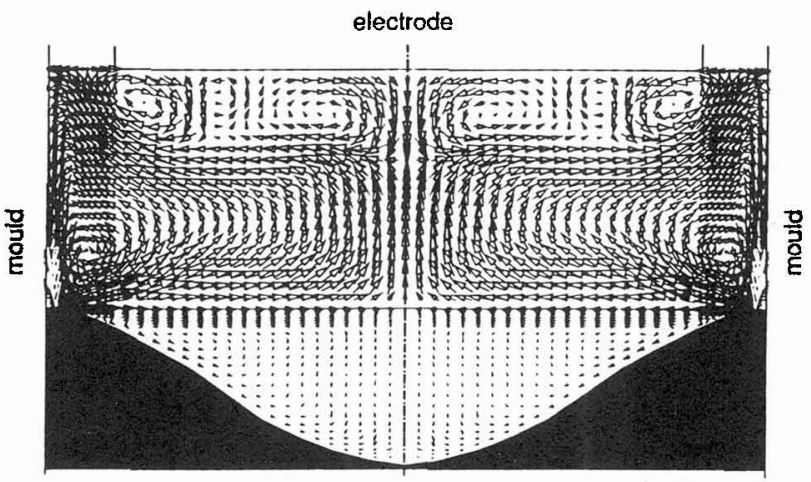

Figure 2 : Electro slag remelting of a chromium steel:calculated movements in the slag and the liquid pool.

The other calculations described in the case of vacuum arc remelting remain possible. Thus, as in the VAR process, it is possible to calculate the distribution of local solidification times in the remelted ingot.

\section{$V$ - ELECTRON BEAM REMELTING}

Over the last decade, the increasing demand for high purity ultra-clean titanium, titanium alloys and superalloys has led to a growing interest in the electron beam remelting process (1). Two variants, employing one or more electron guns, are in use today :

- the discontinuous "Drip Melting" technique, in which a primary ingot is melted by the electron beam(s), the resulting metal droplets forming a secondary ingot which solidifies in a water-cooled copper crucible: 
the continuous "Electron Beam Cold Hearth Refining" process, involving a purification and inclusion separation stage in a cold hearth furnace before ingot formation (figure 3 ).

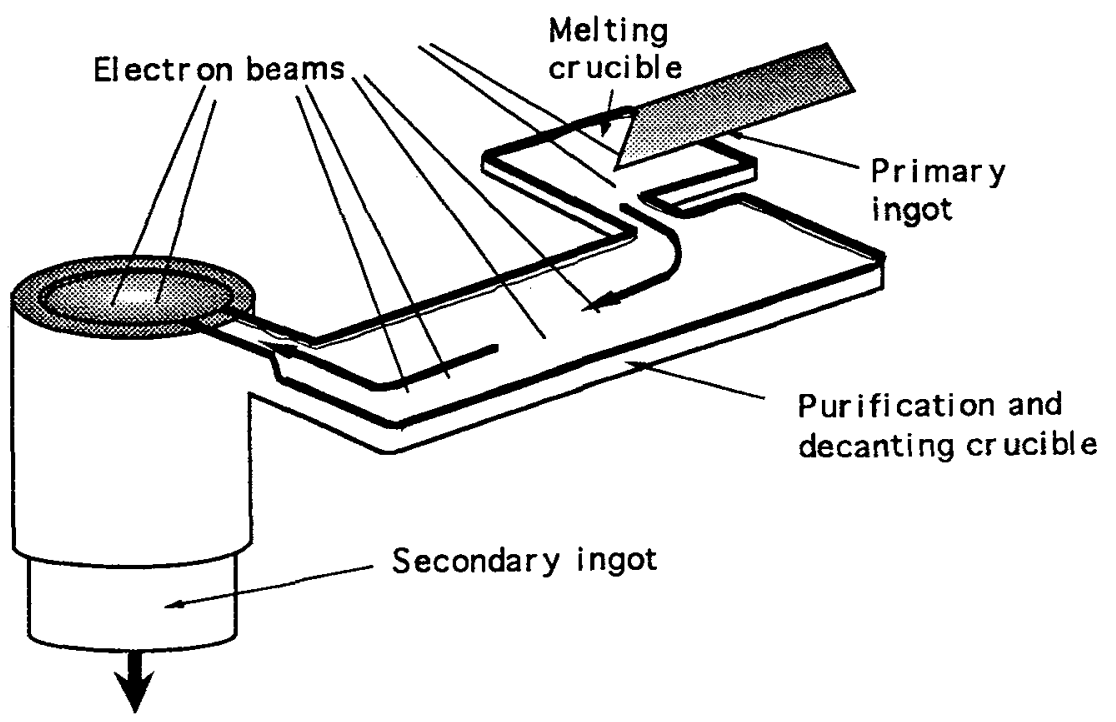

Figure 3 : Schematic diagram showing the principle of the Electron Beam Cold Hearth Refining process.

\section{1) Physical phenomena specific to EB remelting}

The physical phenomena specific to EB remelting are the following :

- At the point of impact of the beam, the kinetic energy of the electrons is converted to heat energy, but the backscattered electrons are responsible for high losses, whose value, which depends on the metal bombarded and the angle of incidence of the beam, represents between 30 and $50 \%$ of the incident energy in the case of titanium.

- The electron beam, which produces a spot of about one centimetre in diameter, sweeps the surface of the liquid metal, combining, for example at the top of the ingot, a rotational movement about the axis of symmetry with a radial movement perpendicular to the axis.

Widely variable energy distributions can therefore be obtained at the surface of the metal.

The high scanning frequency used with modern electron guns enables the energy distribution to be assumed to be quasi-stationary, to a first approximation.

- The temperature gradients in the vicinity of the pool surface, created by the impact of the electron beam, can be extremely high, and can lead to variations in surface tension which give rise to the Marangoni effect.

- Because of the high vacuum in the furnace chamber, volatilization phenomena can be important and cause difficulties in the control of alloy composition.

\section{2) Modelling of the electron beam remelting processes}

As far as the secondary ingot is concerned, models of the electron beam remelting processes have numerous points in common with those for vacuum arc remelting. 
a) Transient thermal model for the secondary ingot is equal to

Contrary to the VAR process, the energy flux density input to the pool surface can be calculated. It

$$
\varphi=\varphi_{E B}-\varphi_{\mathrm{r}}-\varphi_{V}
$$

where $\varphi$ is the net useful heat flux density at the pool surface, $\varphi_{E B}$ is the effective heat flux density supplied by the electron guns, $\varphi_{\mathrm{r}}$ is the heat flux density dissipated by radiation, and $\varphi_{\mathrm{v}}$ is the heat flux density absorbed by volatilization.

Because of this condition, the model gives access to :

- the temperature distribution in the ingot, which can be calculated for widely variable energy distributions.

- the model obviously also gives access to the shape of the liquid pool, and to the distribution of local solidification times.

- the energy balance, shown in figure 4 for a titanium remelting operation, is also obtained. It will be seen that the latent heat of volatilization of titanium is included in the balance.

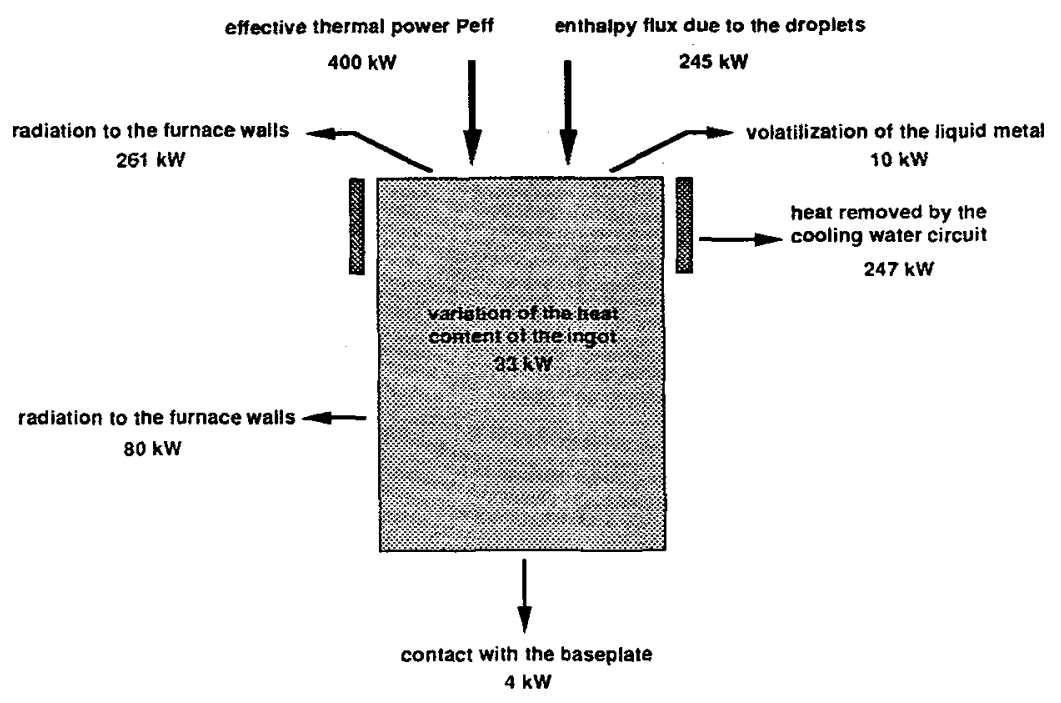

Figure 4 : Thermal balance of a Ti ingot $(0.8 \mathrm{~m}$ diameter,melting rate $550 \mathrm{~kg} / \mathrm{h})$ near the end of an Electron Beam Melting.

\section{b) Coupled transfer model in the melt pool can also be developed}

In an interactive manner, this model solves:

- the Navier-Stokes equations, taking into account natural thermal convection and thermocapillary convection,

- the continuity equation,

- the turbulence equations, based on the $k-\varepsilon$ model,

- the heat balance equation,

- the solute transport equations, which must take into account solute volatilization at the free surface and solute redistribution at the solidification front. 
The results give the velocity and turbulence distributions in the liquid metal. It can be seen that, for an energy flux centered on the mid-radius of the ingot, 2 recirculation vortices exist in the liquid. It is to be noted that the natural convection and thermocapillary forces act in the same direction at the melt surface.

As in the VAR process, the temperature map for the liquid pool reveals considerable stratification.

The solute concentration map, in this case the aluminium content in the liquid titanium, shows the melt pool to be quite homogeneous, except in the vicinity of the surface, principally in the zones overheated by the electron bombardment.

A model of this sort can be used to analyze the influence of operating parameters on the thermal behaviour. The principal parameters of interest are the melting rate, the gun power and the distribution of incident energy on the pool surface.

Their effects can be quantified in terms of the maximum temperature attained, the pool volume and volatilization losses.

Thus, in the case of the remelting of a titanium alloy, it is found that :

- volatilization losses increase with gun power;

- for a given gun power, the losses decrease with increase in melting rate;

- the volatilization losses also depend on the energy distribution at the pool surface (figure 5).

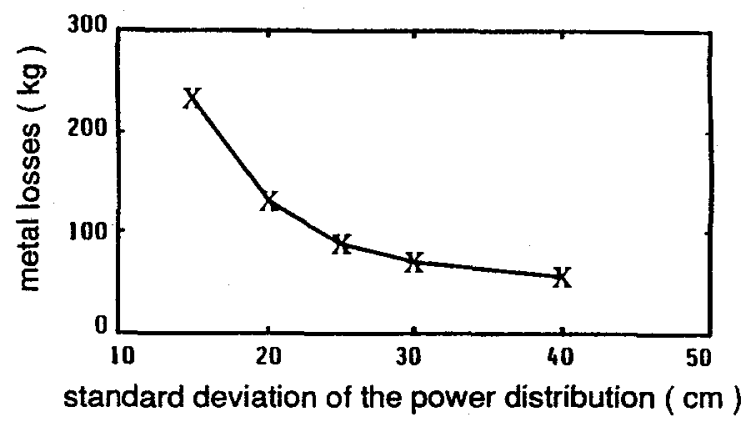

Figure 5 : Influence of power distribution on total metal losses during the EB melting of a Ti ingot.

c) Coupled transfer model for the cold hearth furnace

All of the phenomena previously described must be taken into account, but in this case in the steady state regime. The clearly $3 \mathrm{D}$ geometry leads to a large volume of calculations.

Solidification on the crucible wall is taken into account. A stabilizing effect of the natural convection can be observed, leading to marked temperature stratification.

The metal flow is clearly laminar. The transverse sections reveal recirculation loops due to the natural convection.

The fraction of the metal solidified on the walls is large and represents $80 \%$ of the titanium present in the furnace. The form of the "skull" of solidified metal can be calculated from the model (figure 6).

A similar calculation can also be made with a non-uniform surface energy distribution, which is the usual case in practice. 


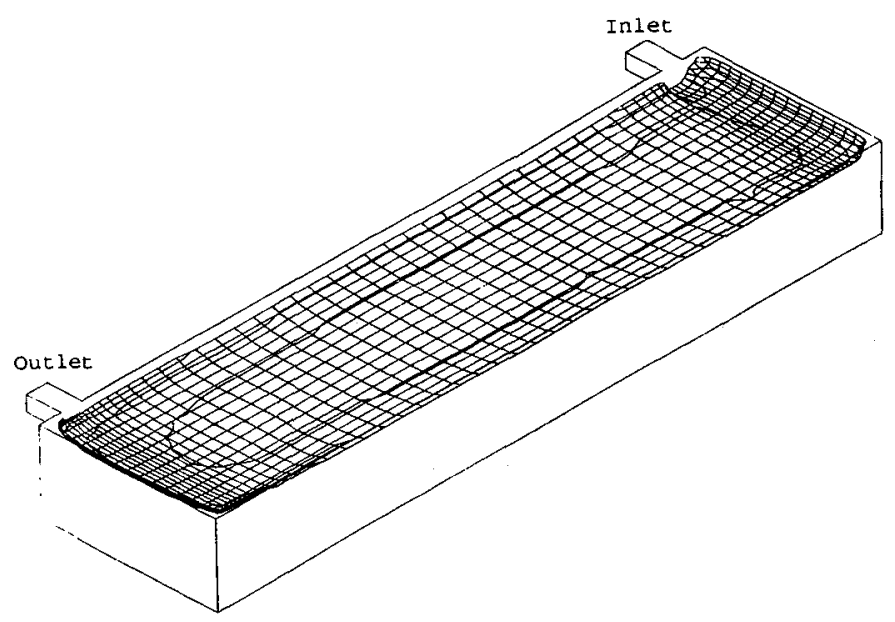

Figure $6:$ EBCHR:Form of the "skull" of solidified titanium calculated from the model with a uniform surface energy distribution equal to $1000 \mathrm{~kW} / \mathrm{m} 2$.

\section{d) Simulation of the trajectory of inclusions and their dissolution rate}

The simulation of the trajectory of inclusions and their dissolution rate is obviously of great interest in the case of electron beam remelting.

Indeed, one of the reasons often invoked to justify the use of electron beam furnaces is their potential for retaining or dissolving inclusions. In the case of titanium alloys, these inclusions are essentially tungsten carbides or titanium oxy-nitrides ("hard- $\alpha$ ").

It is thus interesting to calculate the trajectory of an inclusion, which is given by the fundamental dynamics equation:

$$
\rho_{p} V_{p} \frac{d \vec{u}}{d t}=\vec{P}+\vec{A}+\vec{F}
$$

where $\rho_{p}$ is the density of the inclusion particle, $V_{p}$ is its volume, $u$ is its relative velocity with respect to the fluid, $\mathrm{P}$ is its weight, $\mathrm{A}$ is the Archimedes thrust and $\mathrm{F}$ is the resultant of the actions of the fluid on the inclusion.

Consider for example a "hard- $\alpha$ " type inclusion. During the melting operation, this inclusion will gradually dissolve in the liquid titanium. According to the equilibrium diagram, an $\alpha$ (TiN) phase will be observed surrounded by $\beta(\beta-\mathrm{Ti}+\mathrm{N})$ phase. A dissolution model can be employed to calculate the dimension of each of these phases at any instant.

Coupling of this dissolution model with the calculation of the inclusion trajectory then enables the efficiency of the process to be determined.

For example, the trajectory in liquid titanium of a "hard- $\alpha$ " particle, 500 microns in diameter, is seen to be slightly affected by the recirculatory movements of the metal .

For an inclusion $1000 \mu \mathrm{m}$ in diameter, the particle is seen to become trapped at the solidification front after $22 \mathrm{~s}$, before complete dissolution. 


\section{VI - CONCLUSIONS}

The physical phenomena involved in melting and remelting operations are numerous, complex, and often mutually interactive.

A phenomenological approach coupled with numerical simulation then provides a precious tool, which helps to understand the coupled phenomena and enables optimization of process parameters in order to obtain the required product quality.

Present day computer systems are sufficiently powerful to envisage coupling of macroscopic and microscopic aspects.

However, it is essential not to forget the need to acquire the basic knowledge of the mechanisms involved, to measure the physical-chemical parameters used in the calculations, and to validate the results of the models by experiment. 